\title{
Sonography of the iliotibial band: spectrum of findings*
}

\author{
Ultrassonografia do trato iliotibial: espectro de achados
}

\section{Carlos Frederico Arend ${ }^{1}$}

Arend CF. Sonography of the iliotibial band: spectrum of findings. Radiol Bras. $2014 \mathrm{Jan} / \mathrm{Fev}$;7(1):33-37.

Abstract lliotibial band lesions constitute a potential source of discomfort and are frequently confounded with other conditions which cause pain on the lateral aspect of the hip, thigh and knee. Ultrasonography is usually regarded as a first-line imaging modality in the assessment of such conditions because of its excellent diagnostic accuracy, low cost and wide availability. The correct identification of the structure involved in the production of symptoms leads to an appropriate management and to a higher probability of resolution of the clinical complaint. The present article is aimed at reviewing the different sonographic presentations of iliotibial band syndromes.

Keywords: Sonography; Iliotibial band; lliotibial band friction syndrome.

Resu mo Lesões no trato iliotibial constituem potencial fonte de desconforto e são frequentemente confundidas com outras enfermidades causadoras de dor na face lateral do quadril, coxa e joelho. A ultrassonografia é reconhecida como um método de imagem de primeira linha na investigação destas alterações, em virtude da excelente capacidade diagnóstica, baixo custo e ampla disponibilidade. A correta identificação da estrutura geradora dos sintomas resulta em tratamento específico e maior probabilidade de resolução da queixa clínica. O objetivo deste artigo é revisar as diferentes apresentações ultrassonográficas de doença do trato iliotibial.

Unitermos: Ultrassonografia; Trato iliotibial; Síndrome do trato iliotibial.

\section{INTRODUCTION}

The iliotibial band is a dense fibrotic structure that is found exclusively in the human species, originating in the iliac tubercle, extending to the outer aspect of the thigh, where the fascia latae tensor, gluteus maximus and gluteus medius muscles insert ${ }^{(1)}$, and contributing to the postural control and monopodal equilibrium ${ }^{(2)}$. Its distal perigenicular insertion is complex and includes fibers which extend toward the tibial tubercle, as described by Gerdy ${ }^{(2)}$.

The incidence of iliotibial band syndromes is unknown, considering the high number of patients with subclinical lesions. The most frequent presentation is iliotibial band syndrome that is accepted as the most common cause of lateral gonalgia in long-distance athletes ${ }^{(3,4)}$. However, several other less common alterations may also be observed in such structure, both proximally and distally in relation to the usual affected site in cases of distal iliotibial band syndrome. The present study is aimed at reviewing the different sonographic findings of iliotibial band syndrome.

\section{THE ROLE OF ULTRASONOGRAPHY}

Ultrasonography is recognized as a first-line imaging method in the investigation of iliotibial band injuries because of its excellent diagnostic capability, low cost and wide availability $^{(5)}$. Additionally, $10 \mathrm{MHz}$ linear transducers utilized

* Study developed at Radimagem - Diagnóstico por Imagem, Porto Alegre, RS, Brazil.

1. MD, Radiologist, Radimagem - Diagnóstico por Imagem, Porto Alegre, RS, Brazil. Mailing Address: Dr. Carlos Frederico Arend. Avenida Cristóvão Colombo, 1691, Floresta. Porto Alegre, RS, Brazil, 90560-001. E-mail: carlos_arend@hotmail.com.

Received July 30, 2012. Accepted after revision July 12, 2013. as standard devices in many imaging diagnosis centers, allow for an investigation with an immense wealth of details, achieving axial resolution of $150 \mu \mathrm{m}^{(6)}$. For the purposes of comparison, such an axial resolution is three times better than the $469 \mu \mathrm{m}$ obtained with commercially available 1.5 T magnetic resonance imaging apparatuses ${ }^{(7)}$. Theoretically, transducers with frequencies above $10 \mathrm{MHz}$ can generate a much better imaging resolution with less depth penetration. However, as this method is operator-dependent and based on subjective criteria, the emphasis for the sonographic diagnosis should not be put on the transducer frequency, but on the education of the sonographer to recognize the signs of the disease ${ }^{(8)}$.

\section{SCAN TECHNIQUE}

In the authors' routines, the scan is performed with a 5-13 MHz linear transducer, utilizing only one focal zone adjusted for the tract depth. The positioning of the patient varies according to the region of interest. In the assessment of the proximal iliotibial band, the patient is positioned in lateral decubitus over the symptomatic side, with semi-flexed knee; in the assessment of the distal band, dorsal decubitus with extend knee is preferable. The longitudinal plane allows for a more intuitive interpretation as compared with transverse images, except for snapping hip syndrome investigation, and the agreement with the anatomic alteration with the site of greatest pain sensitivity reported by the patient greatly increases the diagnostic reliability. Contrary to what many patients believe, the contralateral assessment is not mandatory for the diagnosis, although it may be useful in dubious cases or in scans performed with educational purposes ${ }^{(5)}$. 


\section{SPECTRUM OF SONOGRAPHIC FINDINGS}

Iliotibial band syndrome manifestations liable to be documented by ultrasonography shall be individually described in the present article. Generally, there is a resistance to consider hypoechogenicity alone as a criterion of disease due to the ubiquitous anisotropy. Most frequently the diagnosis requires a combination of thickening and hypoechogenicity or, eventually, alterations at dynamic scan.

\section{Proximal iliotibial band syndrome}

Proximal iliotibial band syndrome is an uncommon entity caused either by aging or overuse. Thus, it is most common in women because they present with wider pelvic girdle and shorter femur as compared with men, which leads to a greater mechanical overload on the iliotibial band as a stabilizer element during ambulation ${ }^{(9)}$. Clinically, the discomfort tends to be restricted to the iliac tubercle area, worsening with physical activity. At sonographic evaluation focused on the site of pain reported by the patient, the process is detected as a thickening and hypoechogenicity of the structure (Figure 1).

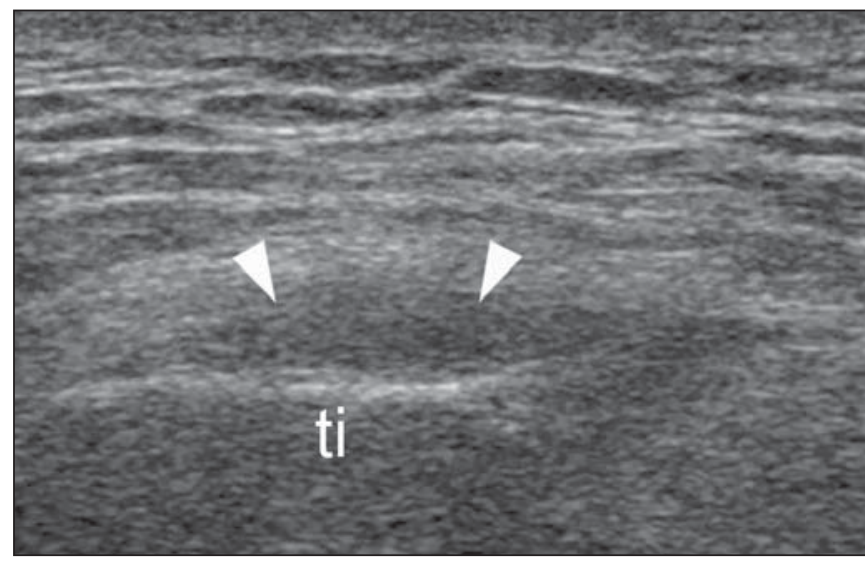

Figure 1. Proximal iliotibial band syndrome. Female, 27-year-old patient, professional athlete, presenting with focal pain adjacent to the right iliac crest that intensifies during physical activity. Longitudinal image acquired with a 5-13 MHz linear transducer demonstrating thickening and hypoechogenicity of the iliotibial band origin (arrow heads) adjacent to the iliac tubercle (it).

\section{Snapping hip syndrome}

The abnormal anterior motion of the iliotibial tract over the greater trochanter of the femur is a common cause of snapping hip syndrome. Reliable statistics on the prevalence of such a condition in the general population are not available, but it is known that it is most common in women at the age range between 15 and 40 years ${ }^{(8)}$. Although usually such snaps do not trigger any symptom, in some cases such abnormality generates disabling pain and a specific diagnosis allows for appropriate treatment and greater chances of resolution of the clinical complaints. At ultrasonography, the patient is assessed with the thigh extended, in lateral decubitus lying on the asymptomatic side, with the transducer transversely positioned over the greater trochanter. In this position, the posterior edge of the iliotibial band and the anterior edge of the gluteus maximus muscle are located posteriorly located in relation to the greater trochanter and slightly displace anteriorly during progressive flexion of the thigh, even in healthy individuals. The diagnostic criterion of such abnormality is not the displacement itself, but the snapping of the structure over the greater trochanter. Pain caused by thigh flexion is the most frequent reason for clinical complaints (Figure 2).
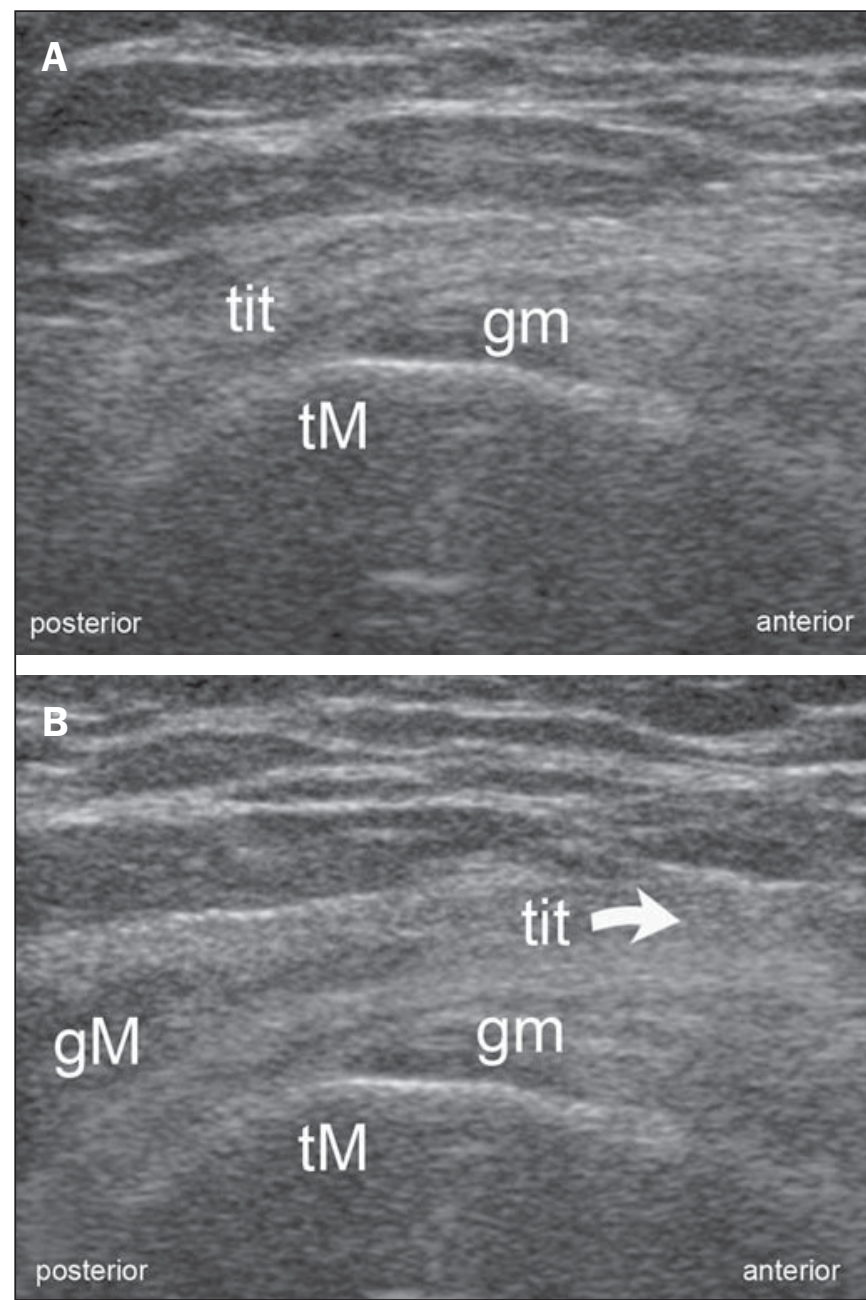

Figure 2. Snapping hip syndrome. Male, 16-year-old, asymptomatic patient referred by his mother for assessment due to chronic snapping in the peritrochanteric region. A: Transverse image acquired with a 5-13 MHz linear transducer, with extended thigh, demonstrates the iliotibial band, the gluteus medius (gm) and the greater trochanter (Gt). Note the posterior edge of iliotibial band located posteriorly to the trochanter. B: With progressive flexion of the thigh, there is a sudden anterior migration of the band and emergence of the gluteus maximus (gM) on the evaluation plane. The arrow indicates the direction of the iliotibial band displacement.

\section{Iliotibial band syndrome}

Iliotibial band syndrome is the most common presentation of iliotibial band disease. It results from overuse that is most common in athletes, particularly marathoners and cyclists $^{(10,11)}$, due to chronic compression of the iliotibial band over a thin layer of fat richly innervated interposed between the iliotibial band and the lateral femoral condyle ${ }^{(12)}$. Anatomical factors, namely, genu varum, excessive internal tibial 
rotation, hip abductor muscle weakness and foot pronation may also contribute to increase the tension over the iliotibial band, predisposing to the syndrome onset ${ }^{(13,14)}$. In older descriptions, the injury was attributed to a cyclic antero-posterior movement of the band over the lateral condyle ${ }^{(15,16)}$, in a mechanism that has, most recently, been questioned. In fact, the iliotibial tract is a distinctive anatomical structure but rather a region of the fascia latae connected with the rough line of the femur by the intermuscular septum, which prevents its anteroposterior motion ${ }^{(17)}$. Thus, currently the most accepted etiopathogenesis of this syndrome is compressive and not frictional ${ }^{(12,17)}$. The typical clinical sign is pain in the region of the lateral femoral condyle, particularly acute at $30^{\circ}$ flexion and exacerbated during the physical examination at local knee motion palpation ${ }^{(18)}$. The compression of the iliotibial band over the fat layer occurs with genicular flexion of approximately $30^{\circ}$, corresponding to the angle at the moment the foot touches the ground and justifies the maximum pain in most patients at that very instant ${ }^{(17)}$.

At imaging, it is easier to start the scan with the transducer longitudinally positioned over the tibial tubercle, where the band insertion can be readily identified. Then, the structure can be proximally followed up to the region of the lateral femoral condyle, where the disease manifestations are concentrated. At its early stage, iliotibial band syndrome manifests as heterogeneity and increased echogenicity of the fat tissue interposed between the band and the femur (Figure 3 ), which evolves with development of contiguous bursa adventitious caused by the chronic compression (Figure 4). The assessment must be performed with mere apposition of the transducer and extended knee, considering that both genicular flexion and the untimely compression of the transducer against the skin surface tend to displace the fluid, making the diagnosis less intuitive (Figure 5). At more advanced stages, thickening and heterogeneity of the iliotibial band itself are observed in the region of the lateral femoral condyle (Figure 6).

\section{Insertional iliotibial band tendinosis}

Insertional iliotibial band tendinosis is considered as a separate entity, located distally to the lateral femoral condyle and with no relation with chronic compression. The physio-

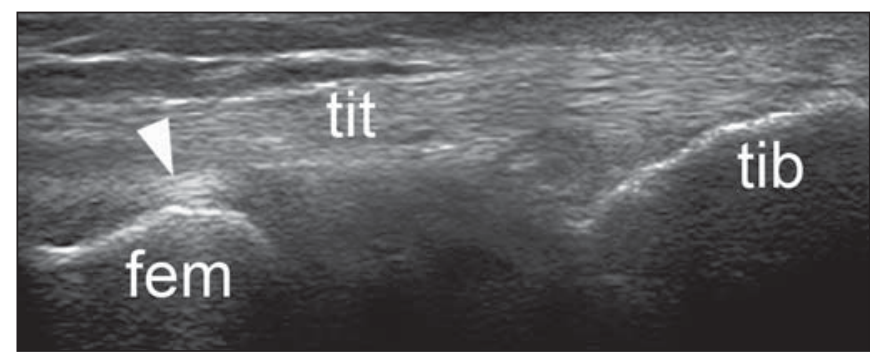

Figure 3. Early stage of iliotibial band syndrome. Male, 37-year-old patient, amateur cyclist, presenting with pain in the lateral aspect of his knee that intensifies during physical activity. Longitudinal image acquired with a 5-13 MHz linear transducer demonstrating increased echogenicity of the fat tissue (arrow head) interposed between the iliotibial band (itb) and the femur (fem). tib, tibia.
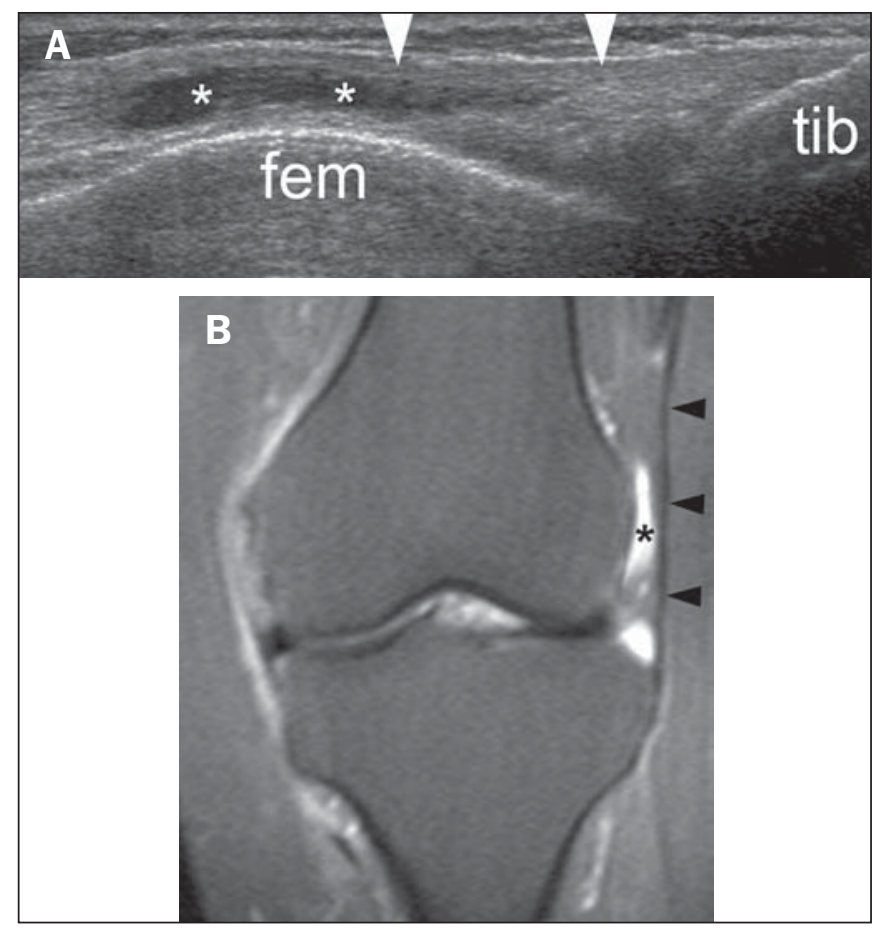

Figure 4. Iliotibial band syndrome. Male, 42-year-old patient, recreational marathoner, presents with intermittent pain in the lateral aspect of his knee, proximally to the joint line. A: Longitudinal image acquired with a 5-13 MHz linear transducer, with extended knee, demonstrates adventitious bursitis in a typical location (asterisks), interposed between the normal iliotibial band (arrow heads) and the lateral femoral (fem) condyle. B: Magnetic resonance imaging - coronal, STIR sequence confirming the sonographic findings. At magnetic resonance imaging, the iliotibial band is identified as an elongated structure with low signal intensity on T1- and T2-weighted sequences, while the neobursa presents a fluid intensity signal. tib, tibia.
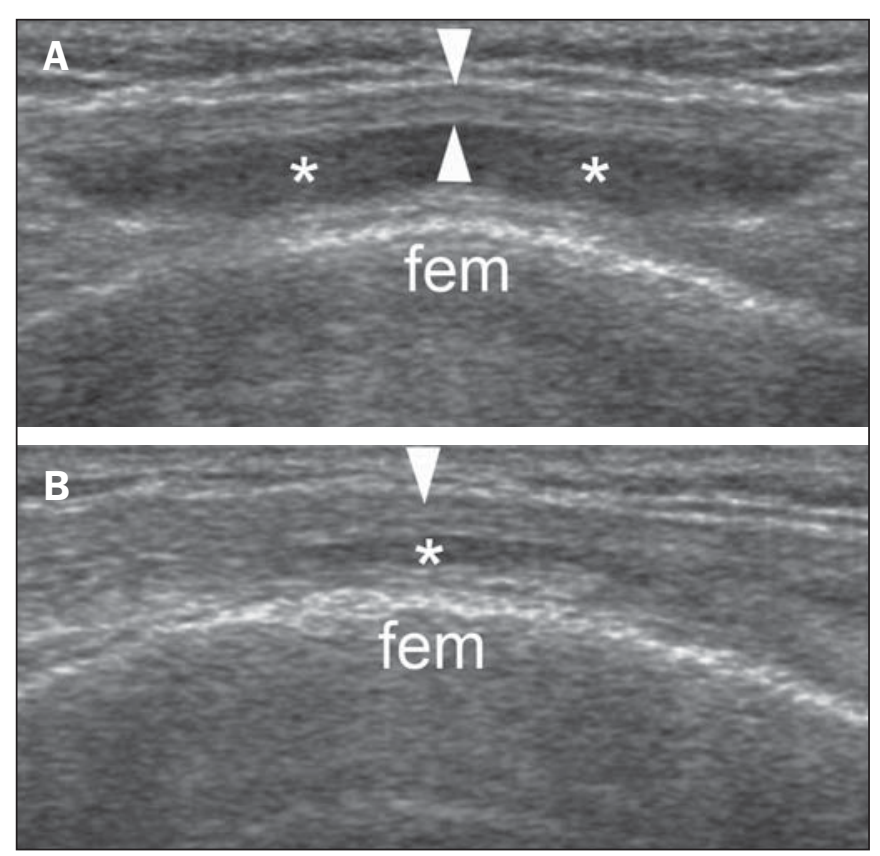

Figure 5. lliotibial band syndrome. Male, 47-year-old patients, recreational marathoner, with constant pain in the lateral aspect of the knee. A: Transverse image acquired with a 5-13 $\mathrm{MHz}$ linear transducer, at the level of the lateral femoral (fem) condyle, with extended knee, demonstrating typical adventitious bursitis (asterisks) deeply located in the normal iliotibial band (arrow heads). B: Compressive study demonstrating bursal walls apposition which may generate false-negative results. 

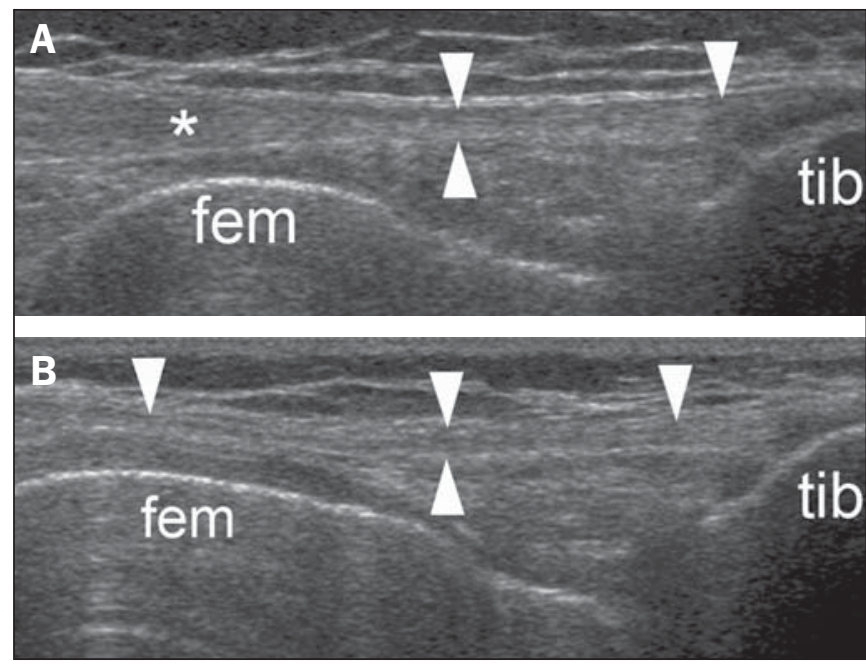

Figure 6. Iliotibial band syndrome. Male, 57-year-old, amateur cyclist, reporting chronic, intermittent pain in the lateral aspect of the knee. A: Longitudinal image acquired with a 5-13 MHz linear transducer, demonstrating thickening and hypoechogenicity of the iliotibial band (asterisk) at the level of the lateral femora (fem) condyle. Also, observe the normal distal band (arrow heads) inserting into the tibia tibia (tib). B: Comparative contralateral image demonstrating the normality along the entire course of the iliotibial band (arrow heads)

pathogenesis of such a condition involves aging and overuse, the latter common in unstable joints due to osteoarthrosis. Innumerable orthopedic misalignments may also increase the tension over the iliotibial band and catalyze the degenerative process over the years. From the clinical point of view, the discomfort epicenter tends to be more distal as compared with the above described iliotibial band syndrome, with eventual proximal irradiation along the lateral aspect of the thigh. The population at risk includes elderly and mid-aged individuals usually sedentary due to the associated genicular osteoarthrosis. At ultrasonography focused on the site of highest sensitivity to pain reported by the patient, insertional tendinosis is seen as thickening and hypoechogenicity of the iliotibial band in the region of the tibia (Figure 7).

\section{The iliotibial band as a catalyst of peritrochanteric syndrome}

Peritrochanteric syndrome is a term utilized to describe a clinical condition characterized by chronic and persistent pain in the region of the greater trochanter of the femur that may be exacerbated by decubitus over the symptomatic side,

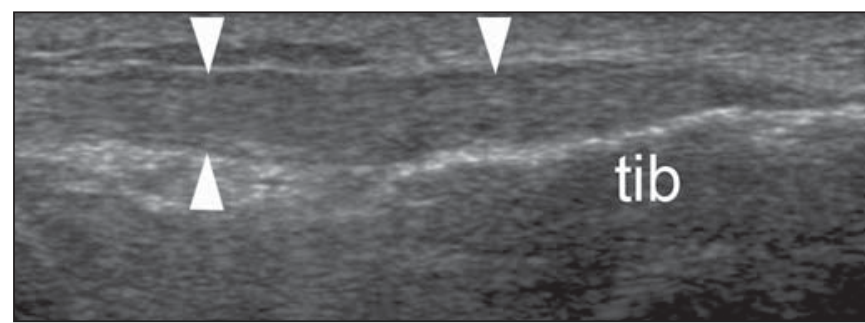

Figure 7. Insertional iliotibial band tendinosis. Male, 89-year-old, sedentary patient, presents with pain epicenter in the lateral aspect of his knee, with proxima irradiation, with no apparent motive. Longitudinal image acquired with a 5-13 $\mathrm{MHz}$ linear transducer, demonstrating juxtainsertional thickening and hypoechogenicity of the iliotibial band (arrow heads) adjacent to the tibia (tib). prolonged orthostatism, high-impact activities, or actions like running, climbing stairs and crossing legs. Pain is more common in women between their fourth and sixth decades of life than in men ${ }^{(19)}$, at a $4: 1$ ratio $^{(20-22)}$. In about $50 \%$ of patients, the pain irradiates over the lateral aspect of the thigh, rarely surpassing the knee ${ }^{(23)}$. Populational studies estimate its prevalence around $10-25 \%$ in industrialized countries ${ }^{(24-}$ 26). In individuals with lumbar pain, the syndrome prevalence increases to $20-35 \%^{(27,28)}$. In the context of primary health care, its incidence is approximately 1.8:1,000 patients/ year ${ }^{(25)}$. Causes are innumerable and include tendon and bursal conditions, and triggering points, only to mention some examples. Additionally, the clinical complaint may be, in truth, a reported pain originating from coxofemoral disease and/or spinal condition. Anamnesis and clinical examination do not allow a safe differentiation amongst the different etiologies, so imaging evaluation is usually indicated to guide the therapy, particularly in cases refractory to the initial management. Historically, iliotibial band syndrome has been preponderantly attributed to a bursal inflammatory process, recognized as trochanteric bursitis. However, with the popularization of imaging methods, the medical professionals have progressively perceived that the main anatomical trigger of symptoms seems to be tendinous rather than bursal, affecting the insertion of the gluteus medius and minimus muscles that are abductors of the lower limb. Because of its anatomical proximity, the iliotibial band may play a significant role in the genesis of the tendon or bursal lesion causing the syndrome due to the chronic friction during ambulation (Figure 8).

\section{CONCLUSION}

Ultrasonography is a rapid, accurate and widely available method capable of demonstrating a wide spectrum of iliotibial band alterations.

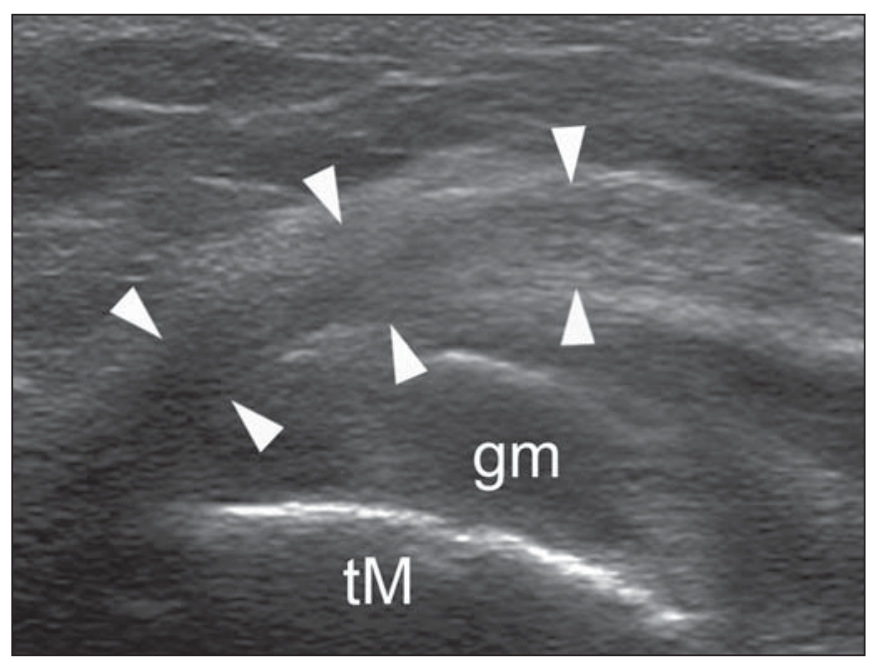

Figure 8. Iliotibial band as a catalyst of peritrochanteric syndrome. Female, 69 year-old, sedentary patient, presenting with chronic peritrochanteric pain. A: Transverse image acquired with a 5-13 $\mathrm{MHz}$ linear transducer demonstrating thickening of the iliotibial band (arrow heads), as well as signs of gluteus medius (gm) tendinosis adjacent to its insertion into the greater trochanter of the femur (Gt). 


\section{REFERENCES}

1. Muhle C, Ahn JM, Yeh L, et al. Iliotibial band friction syndrome: MR imaging findings in 16 patients and MR arthrographic study of six cadaveric knees. Radiology. 1999;212:103-10.

2. Terry GC, Hughston JC, Norwood LA. The anatomy of the iliopatellar band and iliotibial tract. Am J Sports Med. 1986;14:39_ 45.

3. Macera CA, Pate RR, Powell KE, et al. Predicting lower-extremity injuries among habitual runners. Arch Intern Med. 1989;149:25658.

4. Marti B, Vader JP, Minder CE, et al. On the epidemiology of running injuries. The 1984 Bern Grand-Prix study. Am J Sports Med. 1988;16:285-94.

5. Arend CF. MASTER Ultrassonografia musculoesquelética. $2^{\mathrm{a}}$ ed. Rio de Janeiro: Revinter; 2012.

6. Neumann T, Ermert H. Schlieren visualization of ultrasonic wave fields with high spatial resolution. Ultrasonics. 2006;44 Suppl 1: e $1561-6$.

7. Link TM, Majumdar S, Peterfy C, et al. High resolution MRI of small joints: impact of spatial resolution on diagnostic performance and SNR. Magn Reson Imaging. 1998;16:147-55.

8. Arend CF. Top ten pitfalls to avoid when performing musculoskeletal sonography: what you should know before entering the examination room. Eur J Radiol. 2013;82:1933-9.

9. Sher I, Umans H, Downie SA, et al. Proximal iliotibial band syndrome: what is it and where is it? Skeletal Radiol. 201 1;40:1553-6.

10. Khaund R, Flynn SH. Iliotibial band syndrome: a common source of knee pain. Am Fam Physician. 2005;71:1545-50.

11. Fredericson M, Cookingham CL, Chaudhari AM, et al. Hip abductor weakness in distance runners with iliotibial band syndrome. Clin J Sport Med. 2000;10:169-75.

12. Fairclough J, Hayashi K, Toumi H, et al. The functional anatomy of the iliotibial band during flexion and extension of the knee: implications for understanding iliotibial band syndrome. J Anat. 2006;208:309-16.

13. Noble CA. Iliotibial band friction syndrome in runners. Am J Sports Med. 1980;8:232-4.
14. Ferber R, Noehren B, Hamill J, et al. Competitive female runners with a history of iliotibial band syndrome demonstrate atypical hip and knee kinematics. J Orthop Sports Phys Ther. 2010;40:52-8.

15. Holmes JC, Pruitt AL, Whalen NJ. Iliotibial band syndrome in cyclists. Am J Sports Med. 1993;21:419-24.

16. Orchard JW, Fricker PA, Abud AT, et al. Biomechanics of iliotibial band friction syndrome in runners. Am J Sports Med. 1996;24:3759.

17. Fairclough J, Hayashi K, Toumi H, et al. Is iliotibial band syndrome really a friction syndrome? J Sci Med Sport. 2007;10:74-8.

18. Fredericson M, Wolf C. Iliotibial band syndrome in runners: innovations in treatment. Sports Med. 2005;35:451-9.

19. Shbeeb MI, Matteson EL. Trochanteric bursitis (greater trochanter pain syndrome). Mayo Clin Proc. 1996;71:565-9.

20. Anderson TP. Trochanteric bursitis: diagnostic criteria and clinical significance. Arch Phys Med Rehabil. 1958;39:617-22.

21. Krout RM, Anderson TP. Trochanteric bursitis: management. Arch Phys Med Rehabil. 1959;40:8-14.

22. Silva F, Adams T, Feinstein J, et al. Trochanteric bursitis: refuting the myth of inflammation. J Clin Rheumatol. 2008;14:82-6.

23. Gordon EJ. Trochanteric bursitis and tendinitis. Clin Orthop. 1961; 20:193-202.

24. Segal NA, Felson DT, Torner JC, et al. Greater trochanteric pain syndrome: epidemiology and associated factors. Arch Phys Med Rehabil. 2007;88:988-92.

25. Lievense A, Bierma-Zeinstra S, Schouten B, et al. Prognosis of trochanteric pain in primary care. Br J Gen Pract. 2005;55:199-204

26. Collée G, Dijkmans BA, Vandenbroucke JP, et al. Greater trochanteric pain syndrome (trochanteric bursitis) in low back pain. Scand J Rheumatol. 1991;20:262-6.

27. Tortolani PJ, Carbone JJ, Quartararo LG. Greater trochanteric pain syndrome in patients referred to orthopedic spine specialists. Spine J. $2002 ; 2: 251-4$.

28. Collée G, Dijkmans BA, Vandenbroucke JP, et al. A clinical epidemiological study in low back pain. Description of two clinical syn dromes. Br J Rheumatol. 1990;29:354-7. 